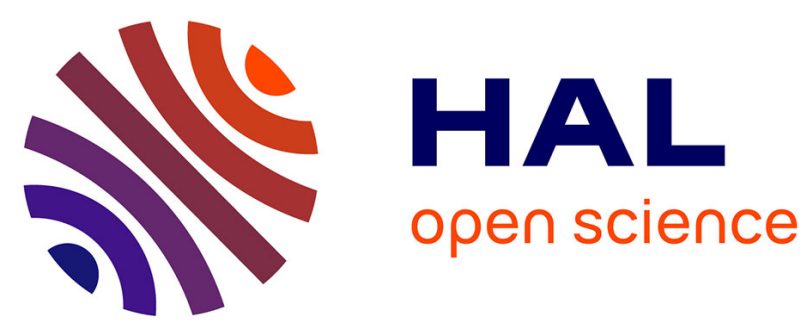

\title{
Homocheletropic Addition of Sulfur Dioxide to 1,4-Dienes. Competition between Cheletropic and Homocheletropic Additions of Polyenes
}

\author{
Jean-Michel Roulet, Brigitte Deguin, Pierre Vogel
}

\section{- To cite this version:}

Jean-Michel Roulet, Brigitte Deguin, Pierre Vogel. Homocheletropic Addition of Sulfur Dioxide to 1,4-Dienes. Competition between Cheletropic and Homocheletropic Additions of Polyenes. Journal of the American Chemical Society, 1994, 116, pp.3639-3640. hal-01927363

\section{HAL Id: hal-01927363 \\ https://hal.science/hal-01927363}

Submitted on 19 Nov 2018

HAL is a multi-disciplinary open access archive for the deposit and dissemination of scientific research documents, whether they are published or not. The documents may come from teaching and research institutions in France or abroad, or from public or private research centers.
L'archive ouverte pluridisciplinaire HAL, est destinée au dépôt et à la diffusion de documents scientifiques de niveau recherche, publiés ou non, émanant des établissements d'enseignement et de recherche français ou étrangers, des laboratoires publics ou privés. 


\section{Homocheletropic Addition of Sulfur Dioxide to 1,4-Dienes. Competition between Cheletropic and Homocheletropic Additions of Polyenes}

Jean-Michel Roulet, Brigitte Deguin, and Pierre Vogel*

Section de Chimie de l'Université 2, rue de la Barre, CH 1005 Lausanne, Switzerland

Homoconjugated dienes can be rearranged into conjugated 1,3-dienes in the presence of $\mathrm{SO}_{2}$ via ene reactions. ${ }^{1,2}$ In the case of norbornadiene (1), which cannot undergo ene reactions with $\mathrm{SO}_{2}, \mathrm{De}$ Lucchi and Lucchini ${ }^{3}$ reported that it adds to $\mathrm{SO}_{2}$ in a $\left(\omega 2_{1}+\pi 2_{s}+\pi 2_{3}\right)$ fashion ${ }^{4}$ to give the corresponding sulfolane 2. In our hands, mixtures of 1 and $\mathrm{SO}_{2}$ led only to polymeric

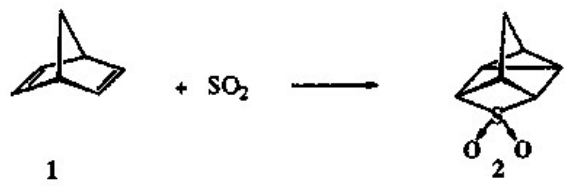

material, unless 2,6-di-tert-butyl-p-cresol was added to the reaction mixture (33\% yield of 2 after 2 days at $25^{\circ} \mathrm{C}$ ). We report here that the homocheletropic addition of $\mathrm{SO}_{2}$ is not restricted only to norbornadiene and that it can compete with the usual cheletropic addition $\left(\omega 2_{1}+\pi^{4}\right)^{4}$ when $\mathrm{SO}_{2}$ is allowed to react with polyenes containing 1,3-diene as well as 1,4-diene moieties. 5

When 3,3-dimethylpenta-1,4-diene $(3)^{6}$ was mixed with 12 13 equiv of $\mathrm{SO}_{2}$ in $\mathrm{CH}_{2} \mathrm{Cl}_{2}$, an $81 \%$ yield of sulfolane $4^{7}$ was obtained after 8 days at $23^{\circ} \mathrm{C}$. Kinetic measurements gave a

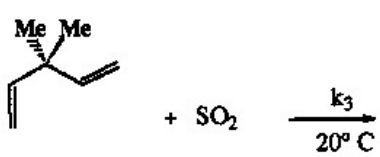

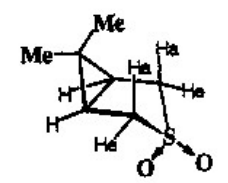

second-order rate constant $k_{3}(296 \mathrm{~K})=1.51 \times 10^{-7} \pm 1.3 \times 10^{-9}$ $\mathrm{dm}^{3} \mathrm{~mol}^{-1} \mathrm{~s}^{-1}$ for reaction $3+\mathrm{SO}_{2} \rightarrow 4$. In the presence of $\mathrm{CF}_{3}$ $\mathrm{COOH}$ ( 0.6 equiv) or $\mathrm{BF}_{3} \cdot \mathrm{Et}_{2} \mathrm{O}$ (0.8 equiv), the rate constants $k_{3}\left(\mathrm{CF}_{3} \mathrm{COOH}, 296 \mathrm{~K}\right)=2.8 \times 10^{-7} \pm 8.7 \times 10^{-9} \mathrm{dm}^{3} \mathrm{~mol}^{-1} \mathrm{~s}^{-1}$ and $k_{3}\left(\mathrm{BF}_{3} \cdot \mathrm{Et}_{2} \mathrm{O}, 298 \mathrm{~K}\right)=7.26 \times 10^{-7} \pm 8.7 \times 10^{-9} \mathrm{dm}^{3} \mathrm{~mol}^{-1}$ $\mathrm{s}^{-1}$, respectively, were measured. This relatively weak accelerating effect due to the protic or Lewis acid sharply contrasts with the

(1) Rogic, M.; Masilamani, D. J. Am. Chem. Soc. 1977, 99, 5219. See also: Capozzi, G.; Lucchini, V.; Marcuzzi, F.; Melloni, G. Tetrahedron Lett. 1980, 21,3289 .

(2) We have found that the reaction of $\mathrm{SO}_{2}$ with penta-1,4-diene in $\mathrm{CH}_{2}$ $\mathrm{Cl}_{2}$ gives mixtures of $(E)$ - and $(Z)$-penta-2,4-dienesulfinic acid and $(E)$ - and (Z)-piperylene.

(3) De Lucchi, O.; Lucchini, V. J. Chem. Soc., Chem. Commun. 1982, 1105.

(4) Woodwand, R. B.; Hoffmann, R. The Conservation of Orbital Symmetry; Acsdemic Press: New York, 1970. Turk, S. D. Cobb, R. L. In 1,4-Cycloadditon Reactions; Hamer, J., Ed.; Academic Press: New York 1967; p 13. Dewar, M. J. S. J. Am. Chem. Sac. 1984, 106, 209.

(S) For three component reactions of $\mathrm{SO}_{2}$, see, e.g.: Decazes, J. M.; Luche, J.-L.; Karan, H. B.; Parthaseratby, R.; Ohrt, J. Tetrahedron Lett. 1972, 3633. Beilus, D Helv. Chem. Acta 1975, 58, 2509.

(6) Eilbracht P. Acker. M. Totzater, W. Chem. Ber 1983, 116, 238.

(7) Data for 4: white crystals; mp 75-76 ${ }^{\circ} \mathrm{C}$; ${ }^{1}$ H NMR (250 MHz, CD CN) $\delta 3.31(\mathrm{~m}, 2 \mathrm{H}, 3 y=14.2 \mathrm{~Hz}, J=6.8 \mathrm{~Hz}, \mathrm{~J}=1.4 \mathrm{H}, \mathrm{H}(2 \mathrm{e}) \mathrm{H}(4 \mathrm{e})$

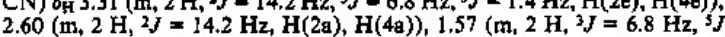
$=1.4 \mathrm{~Hz}, \mathrm{H}(1), \mathrm{H}(5)), 14,0.97(2,6 \mathrm{H}), \mathrm{Me}, \mathrm{in}^{3} \mathrm{CHR}(100.61 \mathrm{MHz}$ $\left.\mathrm{CDCl} l_{3}\right) \delta_{C} 50.6(\mathrm{t}, 1 \mathrm{~J}(\mathrm{C}, \mathrm{H})=143 \mathrm{~Hz}, \mathrm{C}(2), \mathrm{C}(4)), 26.2(\mathrm{q}, 1 \mathrm{~J}(\mathrm{C}, \mathrm{H})=127$ $\mathrm{Hz}$, Me equatorial), 22.2 (d, $1 J(\mathrm{C}, \mathrm{H})=173 \mathrm{~Hz}, \mathrm{C}(1), \mathrm{C}(5)), 21.7(\mathrm{~s}, \mathrm{C}(6))$. $13.7(q, 1 J(C, H)=127 \mathrm{~Hz}$, Me axial $)$.
Scheme 1

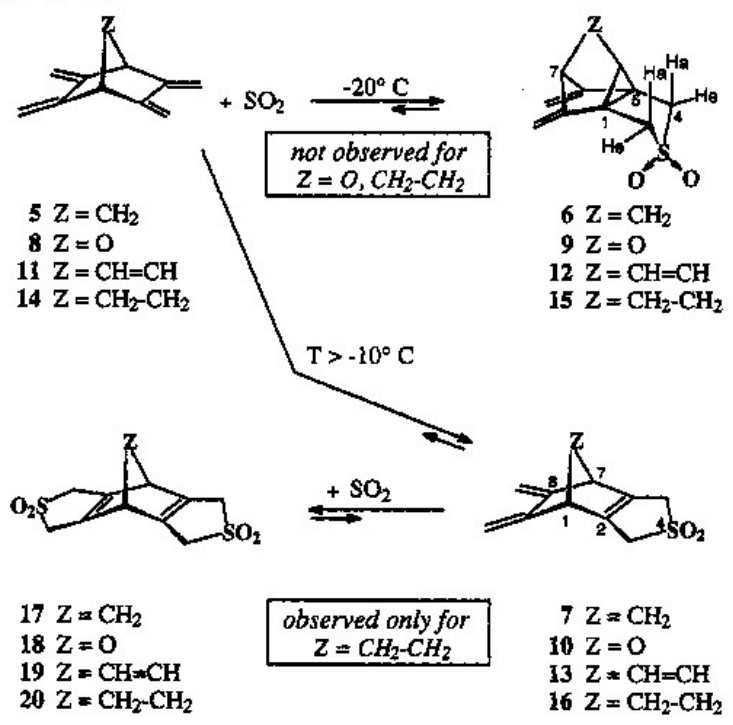

strong acid catalytic effect observed for the hetero-Diels-Alder additions of $\mathrm{SO}_{2}$ to simple 1,3-dienes at low temperature. ${ }^{3}$ The rate of the homocheletropic addition $3+\mathrm{SO}_{2} \rightarrow 4$ was not affected by the presence of a radical scavenger such as 2,6-di-tert-butyl$p$-cresol, thus ruling out the hypothesis that this reaction implies the formation of radicals or diradicals in the rate-determining step.

When 2,3,5,6-tetramethylidenebicyclo[2.2.1] heptane $(5)^{9,10}$ was mixed with $\mathrm{SO}_{2}$ (purified on alkaline alumina (I)) in $\mathrm{CD}_{2} \mathrm{Cl}_{2}$ (sealed NMR tube, $\mathrm{C}_{6} \mathrm{H}_{6}$ as internal reference), the sulfolane $6^{11}$ was formed as a single product at $-20^{\circ} \mathrm{C}$ (Scheme 1). A secondorder rate constant $k_{5}(253 \mathrm{~K})=1.59 \times 10^{-5} \pm 2.6 \times 10^{-7} \mathrm{dm}^{3}$ $\mathrm{mol}^{-1} \mathrm{~s}^{-1}$ was measured. At $0^{\circ} \mathrm{C}, 6$ underwent slow cycloreversion into $5+\mathrm{SO}_{2}$ and then formed sulfolene $7 .{ }^{12}$ In this case, the homocheletropic addition $5+\mathrm{SO}_{2} \rightarrow 6$ is kinetically favored but thermodynamically disfavored compared with the cheletropic addition $5+\mathrm{SO}_{2} \rightarrow 7$. At higher temperatures, 6 and 7 were equilibrated with tetraene 5 , showing that 6 and 7 had similar stabilities (ratio of 6/7, 1:4).

When 2,3,5,6-tetramethylidene-7-oxabicyclo[2.2.1]heptane (8) ${ }^{10,13,14}$ was mixed with a large excess of $\mathrm{SO}_{2}$, no product 9 resulting from a homocheletropic addition could be detected between $-30^{\circ} \mathrm{C}$ and $30^{\circ} \mathrm{C}$. Above $-10^{\circ} \mathrm{C}$, slow formation of sulfolene $10^{15}$ was observed.

(8) Deguin, B.; Vogel, P. J. Am. Chem. Soc. 1992, 114,9210, (9) Florey, A.; Vogel, P. Helv. Chem. Acta 1975, 58, 1488; Pilet, O.; Vogel, P. Ibid. 1981, 64,2563

(10) Vogel, P, In Advances in Theoretically Interesting Molecules; Thummel, R. P., Ed.; JAI Press: Greenwich, CT, 1989; Vol. I, pp 201-355. (11) Data for 6: $\mathrm{mp}^{\circ} 1^{\circ} \mathrm{C} \mathrm{dec}$; ${ }^{\mathrm{H}}$ NMR (400 MHz $\mathrm{CD}_{2} \mathrm{Cl}_{2} \mathrm{SO}_{2}-20^{\circ}$ C) $\delta_{\mathrm{H}} 4.95,4.76\left(2 \mathrm{~s}, 4 \mathrm{H}, \mathrm{H} 2 \mathrm{C}-\mathrm{C}(6), \mathrm{H}_{2} \mathrm{C}=\mathrm{C}(10)\right), 3.76(\mathrm{dm}, 2 \mathrm{H}, 2 J=13.7$ $\mathrm{Hz}, \mathrm{H}(2 \mathrm{e}), \mathrm{H}(4 \mathrm{e})), 3.09(\mathrm{dm}, 2 \mathrm{H}, 2 \mathrm{~J}=13.7 \mathrm{~Hz}, \mathrm{H}(2 \mathrm{e}), \mathrm{H}(4 \mathrm{~g})), 2.86(\mathrm{t}, \mathrm{H}$ $\mathrm{Hz}, \mathrm{H}(2 \mathrm{e}), \mathrm{H}(4 \mathrm{e})), 3.09(\mathrm{dm}, 2 \mathrm{H}, 2 J=13.7 \mathrm{~Hz}, \mathrm{H}(2 \mathrm{e}), \mathrm{H}(4 \mathrm{~g})), 2.86(\mathrm{t}, 1 \mathrm{H}$, $3 J=1.4 \mathrm{~Hz}, \mathrm{H}(7)), 2.31\left(\mathrm{t}, 1 \mathrm{H},{ }^{3} \mathrm{~J}=1.0 \mathrm{~Hz}, \mathrm{H}(9)\right), 1.84\left(\mathrm{dd}, 2 \mathrm{H},{ }^{3} \mathrm{~J}=1.4\right.$ $\left.\mathrm{Hz},{ }^{3} \mathrm{~S}=1.0 \mathrm{~Hz}, \mathrm{H}(8)\right) ;{ }^{13} \mathrm{C}$ NMR $\left(100.61 \mathrm{MHz}, \mathrm{CD}_{2} \mathrm{Cl}_{2}, \mathrm{SO}_{2},-20^{\circ} \mathrm{C}\right) \delta_{\mathrm{c}}$ $151.1(\mathrm{~s}, \mathrm{C}(6), \mathrm{C}(10)), 100.1\left(\mathrm{t}, \mathrm{J}(\mathrm{C}, \mathrm{H})=159 \mathrm{~Hz}, \mathrm{H}_{2} \mathrm{C}=\mathrm{C}(6), \mathrm{H}_{2} \mathrm{C}=\mathrm{C}\right.$.

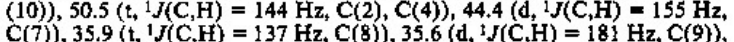
34.7 (s, $\mathrm{C}(1), \mathrm{C}(5))$.

(12) Data for 7: $\mathrm{mp} 96^{\circ} \mathrm{C} \mathrm{dec} ;{ }^{1} \mathrm{H}$ NMR (250 MHz, CDCl $)$ ) $\delta_{\mathrm{H}} 5.26,5.03$ (12) Data for 7: $\mathrm{mp} 96^{\circ} \mathrm{C} \mathrm{dec} ;{ }^{1} \mathrm{H}$ NMR $\left(250 \mathrm{MHz}, \mathrm{CDCl}_{3}\right) \delta_{\mathrm{H}} 5.26,5.03$ $\left(2 \mathrm{~s}, 4 \mathrm{H}, \mathrm{H}_{2} \mathrm{C}-\mathrm{C}(8), \mathrm{H}_{2} \mathrm{C}-\mathrm{C}(9)\right), 3.92\left(\mathrm{dm}, 2 \mathrm{H},{ }^{2} J=16.4 \mathrm{~Hz}, \mathrm{H}(3 \mathrm{e})\right.$,
$\mathrm{H}(\mathrm{se})), 3.65\left(\mathrm{dm}, 2 \mathrm{H},{ }^{\mathrm{j}}=16.4 \mathrm{~Hz}, \mathrm{H}(3 \mathrm{a}), \mathrm{H}(5 \mathrm{a}), 3.44(\mathrm{t}, 2 \mathrm{H}, 3 \mathrm{~J}=1.7\right.$ $\mathrm{H}(\mathrm{Se})), 3.65$ (dm, $2 \mathrm{H}, 2 \mathrm{~J}=16.4 \mathrm{~Hz}, \mathrm{H}(3 \mathrm{a}), \mathrm{H}(5 \mathrm{a})), 3.44(\mathrm{t}, 2 \mathrm{H}, 3 \mathrm{~J}=1.7$ $\mathrm{Hz}, \mathrm{H}(1), \mathrm{H}(\mathrm{l})), 2.01\left(\mathrm{dt}, 1 \mathrm{H},{ }^{2 J}=8.7 \mathrm{~Hz},{ }^{3 J}=1.7 \mathrm{~Hz}, \mathrm{H}(10)\right), 1,80(\mathrm{dt}$,
$\left.\mathrm{IH},{ }^{3} J=1.7 \mathrm{~Hz},{ }^{2} J=8.7 \mathrm{~Hz}, \mathrm{H}(10)\right) ;{ }^{3} \mathrm{C} \mathrm{NMR}\left(100.61 \mathrm{MHz}, \mathrm{CD}_{2} \mathrm{Cl}_{2}\right) \delta_{\mathrm{C}}$ $\left.1 \mathrm{H},{ }^{3} J=1.7 \mathrm{~Hz},{ }^{2} J=8.7 \mathrm{~Hz}, \mathrm{H}(10)\right) ;{ }^{13} \mathrm{C} \mathrm{NMR}\left(100.61 \mathrm{MHz}, \mathrm{CD}_{2} \mathrm{Cl}_{2}\right){ }_{\mathrm{C}}$
$147.2(\mathrm{C})$ $147.2(\mathrm{~s}, \mathrm{C}(8), \mathrm{C}(9)), 140.9(\mathrm{~s}, \mathrm{C}(2), \mathrm{C}(6)), 104.6(\mathrm{t}, 1 J(\mathrm{C}, \mathrm{H})=159 \mathrm{~Hz}$,
$\left.\mathrm{H}_{2} \mathrm{C}=\mathrm{C}(8), \mathrm{H}_{2} \mathrm{C}=\mathrm{C}(9)\right), 56.4(\mathrm{t}, \mathrm{JJ}(\mathrm{C}, \mathrm{H})=145 \mathrm{~Hz}, \mathrm{C}(3), \mathrm{C}(5)), 51.7(\mathrm{~d}$, $J(\mathrm{C}, \mathrm{H})=145 \mathrm{~Hz}, \mathrm{C}(1), \mathrm{C}(7)), 49.3(\mathrm{t}, 1 J(\mathrm{C}, \mathrm{H})=137 \mathrm{~Hz}, \mathrm{C}(10))$.

(13) Vogel, P.; Florey, A. Helv. Chem. Acta 1974, 97, 200. Mahaim, C.; Carrupt, P.-A.; Hagenbuch, J.-P.; Florey, A.; Vogel, P. Ibid. 1980, 63, 1149. 
Between $-40^{\circ} \mathrm{C}$ and $25^{\circ} \mathrm{C}, \mathrm{SO}_{2}$ was added to $5,6,7,8$-tetramethylidenebicyclo[2.2.2] ture of sulfolane $12^{17}$ and sulfolene 13. ${ }^{18}$ This product ratio and the rate of this reaction $\left(k_{11}(253 \mathrm{~K})=4.20 \times 10^{-6} \pm 6.3 \times 10^{-8}\right.$ $\mathrm{dm}^{3} \mathrm{~mol}^{-1} \mathrm{~s}^{-1}$ ) were not affected significantly by the presence of 2,6-di-tert-butyl-p-cresol or $\mathrm{CF}_{3} \mathrm{COOH}$. Above $25^{\circ} \mathrm{C}, 12$ was slowly isomerized into 13 via homocheletropic elimination, giving $11+\mathrm{SO}_{2}$, which underwent cheletropic addition to 13,13 being more stable than 12. Heating of 13 above $100^{\circ} \mathrm{C}$ led to elimination of $\mathrm{SO}_{2}$ and recovery of pentaene 11. In contrast. tetraene $14^{10,16}$ did not to give any trace of the expected sulfolane 15 when treated with various amounts of $\mathrm{SO}_{2}$ between $-40^{\circ} \mathrm{C}$ and $40^{\circ} \mathrm{C}$. The sulfolene 16 was formed instead $\left(k_{14}(253 \mathrm{~K})\right.$ $=1.75 \times 10^{-5} \pm 5.5 \times 10^{-7} \mathrm{dm}^{3} \mathrm{~mol}^{-1} \mathrm{~s}^{-1}$ ), which added, above $-20^{\circ} \mathrm{C}$, a second equivalent of $\mathrm{SO}_{2}$ to give the bis-sulfolene $20.1^{19}$ Below $-65^{\circ} \mathrm{C}, 14$ was equilibrated slowly with a $3: 2$ mixture of two diastereomeric sultines resulting from the hetero-Diels-Alder addition of $\mathrm{SO}_{2}{ }^{8}$

In contrast with the facile reaction $16+\mathrm{SO}_{2} \rightarrow 20$, no trace of the corresponding bis-sulfolenes 17,18 , and 19 resulting from the cheletropic additions of $\mathrm{SO}_{2}$ to 7,10 , and 13 could be detected

(14) For synthetic applications of this tetraene, see, e.g.: Tamariz, J.; Vogel, P. Tetrahedron 1984, 40, 4549. Tornare, J.-M.; Vogel, P. Helv. Chem. Acta 1985, 68, 1069. Ashton, P. R.; Isaacs, N. S.; Kohnke, F. H.; Mathias, J. P.; Stoddat, J. F. Angew, Chem. Int. Ed. Engl. 1989, 28, 1258. Ashton, P. R.; Girreser. U.; Giuffrida, D.; Kohnke, F. H;; Mathias, J. P.; Raymo, F. M; Slawin, A. M. Z.; Stoddart, J. F.; Williams, D. J. J. Am. Chem. Soc. 1993,
115,5422 .

(15) Data for 10: mp $103^{\circ} \mathrm{C} \mathrm{dec} ;{ }^{1} \mathrm{H}$ NMR (250 MHz, $\left.\mathrm{CDCl}_{3}\right) \delta_{\mathrm{H}} 5.38$, , $\left(\mathrm{dm}, 2 \mathrm{H},{ }^{2 J}=16.5 \mathrm{~Hz}, \mathrm{H}(3 \mathrm{e}), \mathrm{H}(\mathrm{Se})\right), 3.72\left(\mathrm{dm}, 2 \mathrm{H},{ }^{2 J}=16.5 \mathrm{~Hz}, \mathrm{H}(3 \mathrm{a})\right.$, $\mathrm{H}(5 \mathrm{a})) ;{ }^{13} \mathrm{C}$ NMR $\left(100.61 \mathrm{MHz}, \mathrm{CDCl}_{3}\right) \delta_{\mathrm{C}} 141.0(\mathrm{~s}, \mathrm{C}(8), \mathrm{C}(9)), 140.4(\mathrm{~s}$, $\mathrm{C}(2), \mathrm{C}(6)), 104.5\left(\mathrm{t}, 1 \mathrm{~J}(\mathrm{C}, \mathrm{H})=161 \mathrm{~Hz}, \mathrm{H}_{2} \mathrm{C}=\mathrm{C}(8), \mathrm{H}_{2} \mathrm{C}=\mathrm{C}(9)\right), 83.0(\mathrm{~d}$,

$C, H)=163 \mathrm{~Hz}, \mathrm{C}(1), \mathrm{C}(7)), 55.1(\mathrm{t}, 1 \mathrm{~J}(\mathrm{C}, \mathrm{H})=144 \mathrm{~Hz}, \mathrm{C}(3), \mathrm{C}(5))$.

(16) Chollet, A.; Wismer, M.; Vogel, P. Tetrahedron Lett. 1976, 47, 4271.

(17) Pure sulfolane 12 was obtained by allowing 13 to react with $N$-phenyltriazolinedione at $-20^{\circ} \mathrm{C}$. The Diels-Alder adduct so obtained was readily separated from 12 by column chromatography on silica gel. Data for 12: mp $122^{\circ} \mathrm{C}$ dec; ${ }^{1} \mathrm{H}$ NMR $\left(250 \mathrm{MHz}, \mathrm{CDCl}_{3}\right) \delta_{\mathrm{H}} 6.13$ (ddd, $1 \mathrm{H},{ }^{3} \mathrm{~J}=$ $7.8,7.0 \mathrm{~Hz}, 4 J=2.8 \mathrm{~Hz}, \mathrm{H}(8)), 5.98(d \mathrm{dd}, 1 \mathrm{H}, 3 \mathrm{~J}=7.8,5.5 \mathrm{~Hz}, 4 J=1.5$ $\mathrm{Hz}, \mathrm{H}(9)), 5.02,4.70\left(2 \mathrm{~s}, 4 \mathrm{H}, \mathrm{H}_{2} \mathrm{C}=\mathrm{C}(6), \mathrm{H}_{2} \mathrm{C}=\mathrm{C}(1 i)\right), 3.78\left(\mathrm{~d}, 2 \mathrm{H},{ }^{2} J\right.$ - $13.5 \mathrm{~Hz}, \mathrm{H}(2 \mathrm{e}), \mathrm{H}(4 \mathrm{e})), 3.48(\mathrm{~d}, 1 \mathrm{H}, 3 \mathrm{~J}=7.0 \mathrm{~Hz}, 4 J=1.5 \mathrm{~Hz}, \mathrm{H}(7)), 3.23$ $(\mathrm{d}, 2 \mathrm{H}, 2 J=13.5 \mathrm{~Hz}, \mathrm{H}(2 \mathrm{a}), \mathrm{H}(4 \mathrm{a})), 2.62$ (dd, $1 \mathrm{H}, 3 f=5.5 \mathrm{~Hz}, 4 J=2.8$ $\mathrm{Hz}, \mathrm{H}(10)) ;{ }^{13} \mathrm{C}$ NMR $\left(100.61 \mathrm{MHz}, \mathrm{CD}_{2} \mathrm{C}, \delta_{\mathrm{C}} 145.2\right.$ (s, C(6), C(11)), $129.8(\mathrm{~d}, 1 \mathrm{~J}(\mathrm{C}, \mathrm{H})=173 \mathrm{~Hz}, \mathrm{C}(8)), 122.2(\mathrm{~d}, 1 \mathrm{~J}(\mathrm{C}, \mathrm{H})=169 \mathrm{~Hz}, \mathrm{C}(9)), 102.8$ $\left(\mathrm{t}, \mathrm{J} J(\mathrm{C}, \mathrm{H})=159 \mathrm{~Hz}, \mathrm{H}, \mathrm{C}=\mathrm{C}(6), \mathrm{H}_{2} \mathrm{C}=\mathrm{C}(11)\right), 53,7(\mathrm{t}, \mathrm{J}(\mathrm{C}, \mathrm{H})=143 \mathrm{~Hz}$, $\mathrm{C}(2), \mathrm{C}(4)), 50.2\left(\mathrm{~d},{ }^{1} J(\mathrm{C}, \mathrm{H})=134 \mathrm{~Hz}, \mathrm{C}(7)\right), 39.7(\mathrm{~d}, 1 J(\mathrm{C}, \mathrm{H})=171 \mathrm{~Hz}$, C(10)), $36.6(\mathrm{~s}, \mathrm{C}(1), \mathrm{C}(5))$

(18) Data for 13: $\mathrm{mp}>145^{\circ} \mathrm{C}$ dec; ${ }^{1} \mathrm{H}$ NMR $\left(250 \mathrm{MHz}, \mathrm{CDCl}_{3}\right) \delta_{\mathrm{c}} 6.48$ (dd $2 \mathrm{H}, 3 J=4.5 \mathrm{~Hz}, J=3.2 \mathrm{~Hz}, \mathrm{H}(10), \mathrm{H}(11)), 5.20,4.93(2 \mathrm{~s}, 4 \mathrm{H}$

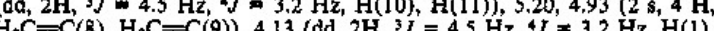
$\left.\mathrm{H}_{2} \mathrm{C}=\mathrm{C}(8), \mathrm{H}_{2} \mathrm{C}=\mathrm{C}(9)\right), 4.13$ (dd, $2 \mathrm{H}, 3 \mathrm{~J}=4.5 \mathrm{~Hz}_{3}, 2=3.2 \mathrm{~Hz}, \mathrm{H}(1)$, (7) $(8), C(9)) 1348(\mathrm{~s}, \mathrm{C}(2) \mathrm{C}(6)) 1333(\mathrm{~d}, \mathrm{JC}(\mathrm{C})=173 \mathrm{H}) \mathrm{C}(10)$ $(\mathrm{s}, \mathrm{C}(8), \mathrm{C}(9)), 134.8(\mathrm{~s}, \mathrm{C}(2), \mathrm{C}(6)), 133.3(\mathrm{~d}, \mathrm{~J}(\mathrm{C}, \mathrm{H})=173 \mathrm{~Hz}, \mathrm{C}(10)$,

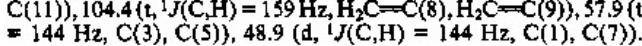

between $0^{\circ} \mathrm{C}$ and $100^{\circ} \mathrm{C}$ when polyenes 5,8 , and 11 , respectively, were allowed to react with $\mathrm{SO}_{2}$ for several days. This is probably due to the fact that the corresponding bis-sulfolenes 17,18 , and 19 are unstable above $0{ }^{\circ} \mathrm{C}$ with respect to the products of cheletropic elimination because of the extra strain of bicyclo[2.2.1] hepta-2,5-diene (17), 7-oxabicyclo[2.2.1] hepta-2,5-diene (18), and barrelene systems (19) compared with bicyclo[2.2.2]octa-2,5-diene derivatives such as $\mathbf{2 0 . 1 0 , 2 0}$

The homocheletropic additions $5+\mathrm{SO}_{2} \rightarrow 6$ and $11+\mathrm{SO}_{2}$ $\rightarrow 12$ (occurring at $-20^{\circ} \mathrm{C}$ ) were significantly faster than reaction $3+\mathrm{SO}_{2} \rightarrow 4$ (occurring at $20^{\circ} \mathrm{C}$ ). This might be explained in terms of an electronic factor: the olefinic moieties in polyenes 5 and 11 being part of $s$-cis-butadiene units are more polarizable than those of the simple 1,4-diene 3. Differential electronic effects could also be responsible for the competition between the cheletropic and homocheletropic additions of $\mathrm{SO}_{2}$ to polyenes $\mathbf{5}$, 8, 11, and 14. Alternatively, geometrical factors such as the angle between the two reacting olefinic moieties and the distance between them could also be decisive.

Work is underway in our laboratory to put these hypotheses on firmer ground. For the moment it is interesting to realize that a simple compound such as $\mathrm{SO}_{2}$ has a wealth of chemistry to offer as a cycloaddend.

Acknowledgment. We are grateful to the Swiss National Science Foundation, the Fonds Herbette (Lausanne), and F. Hoffmann-La Roche AG \& Co. (Basel) for financial support.

Supplementary Material Available: Preparations and characteristics of sulfolanes 6,12 , sulfolenes $7,10,13,16$, bissulfolene 20, and sultines $21,21^{\prime}$ resulting from the hetero-Diels-Alder addition of $\mathrm{SO}_{2}$ to 14; kinetic measurements of the cycloaddition of $\mathrm{SO}_{2}$ on 3 and 11, (14 pages). This material is contained in many libraries on microfiche, immediately follows this article in the microfilm version of the journal, and can be ordered from the ACS; see any current masthead page for ordering information.

(19) Data for 16: $\mathrm{mp} 115^{\circ} \mathrm{C}$ doc; ' $\mathrm{H}$ NMR (250 MHz, CDCl $){ }^{8} \mathrm{~h} 5.23$ $4.84\left(2 \mathrm{~s}, 4 \mathrm{H}, \mathrm{H}_{3} \mathrm{C}=\mathrm{C}(8), \mathrm{H}_{2} \mathrm{C}=\mathrm{C}(9)\right), 3.91(\mathrm{~s}, 4 \mathrm{H}, \mathrm{H}(3), \mathrm{H}(5)), 3.27(\mathrm{~b}$ s, $2 \mathrm{H}, \mathrm{H}(1), \mathrm{H}(7)), 1.79,1.56(2 \mathrm{~m}, 4 \mathrm{H}, \mathrm{H}(10), \mathrm{H}(11))$, ${ }^{13} \mathrm{C} \mathrm{NMR}(100.6$ $\mathrm{MHz}, \mathrm{CDCl})_{3} \delta_{\mathrm{C}} 144.7(\mathrm{~s}, \mathrm{C}(8), \mathrm{C}(9)), 133.9(\mathrm{~s}, \mathrm{C}(2), \mathrm{C}(6)), 104.5(\mathrm{t}, 1 \mathrm{~J}(\mathrm{C}, \mathrm{H})$ $\left.=159 \mathrm{~Hz}, \mathrm{H}_{2} \mathrm{C}=\mathrm{C}(8), \mathrm{H}_{2} \mathrm{C}=\mathrm{C}(9)\right), 57.8(\mathrm{t}, \mathrm{I}(\mathrm{C}, \mathrm{H})=144 \mathrm{~Hz}, \mathrm{C}(3), \mathrm{C}(5))$ $42.7(\mathrm{~d}, 1 J(\mathrm{C}, \mathrm{H})=140 \mathrm{~Hz}, \mathrm{C}(1), \mathrm{C}(\mathrm{7})), 26.1(\mathrm{t}, \mathrm{IJ}(\mathrm{C}, \mathrm{H})=134 \mathrm{~Hz}, \mathrm{C}(10)$ C(1i)) Data for 20: $\mathrm{mp}>145^{\circ} \mathrm{C}$ dec i ${ }^{1} \mathrm{NMR}(250 \mathrm{MHz} \mathrm{CD} \mathrm{CN})$ 3.87 (br s, $8 \mathrm{H}, \mathrm{H}(3), \mathrm{H}(5), \mathrm{H}(9), \mathrm{H}(11)), 3.70(\mathrm{br} \mathrm{s}, 2 \mathrm{H}, \mathrm{H}(1), \mathrm{H}(7)), 1.52$ (s, $\left.4 \mathrm{H}, \mathrm{H}(13), \mathrm{H}(14)\} ;{ }^{13} \mathrm{C} \mathrm{NMR}(100.6) \mathrm{MHz}, \mathrm{CD}_{3} \mathrm{CN}\right)$ oc $135.3(\mathrm{~s}, \mathrm{C}(2)$ C(6), $\mathrm{C}(8), \mathrm{C}(12)), 57.8(\mathrm{t}, 1 \mathrm{C}(\mathrm{H})=145 \mathrm{~Hz}, \mathrm{C}(3) \mathrm{C}(5), \mathrm{C}(9), \mathrm{B}(1 \mathrm{~s})$ $38.5(\mathrm{~d}, 1 J(\mathrm{C}, \mathrm{H})=144 \mathrm{~Hz}, \mathrm{C}(1), \mathrm{C}(7)), 25.6(\mathrm{t}, 1 J(\mathrm{C}, \mathrm{H})=128 \mathrm{~Hz}, \mathrm{C}(13)$ C(14)).

(20) Turner, R. B. J. Am. Chem. Soc. 1964, 86, 3586. Jensen, J. L. Prog. Phys. Org. Chem. 1976, I2, 189. 